\title{
Synthesis some 4-substituted 9,10-anthraquinones
}

Vasyl I. Shupeniuk ${ }^{\mathrm{a}}$, Tetyana M. Taras ${ }^{\mathrm{a}}$, Oksana P. Sabadakh ${ }^{\mathrm{a}}$, Eugene R. Luchkevich ${ }^{\mathrm{a}}$, Yurii Eu. Kornii ${ }^{b}$

${ }^{a}$ Department of Chemistry of the Environment and Chemical Education, Vasyl Stefanyk

Precarpathian National University, Shevchenko Street 57, Ivano-Frankivsk 76018, Ukraine

${ }^{b}$ Department of Chemistry of Bioactive Nitrogen-Containing Heterocyclic Bases, V.P. Kukhar Institute of Bioorganic Chemistry and Petrochemistry, NAS of Ukraine, Murmanska st., 1, Kyiv, 02094, Ukraine

vasyl.shupeniuk@pnu.edu.ua

Keywords: Bromaminic acid, Ullmann reaction, LC-MS, 4-substituted 9,10-anthraquinones, diethanolamine.

New 4-substituted 9,10-anthraquinones (6 compouds) with amino derivations fragments were synthesized through the substitution of the bromaminic acid by amines using the Ullmann coupling reaction. The structures of the synthesized compounds were determined using LC-MS, ${ }^{1} \mathrm{H}$ NMR, ${ }^{13} \mathrm{C}$ NMR spectroscopy, and elemental analysis data.

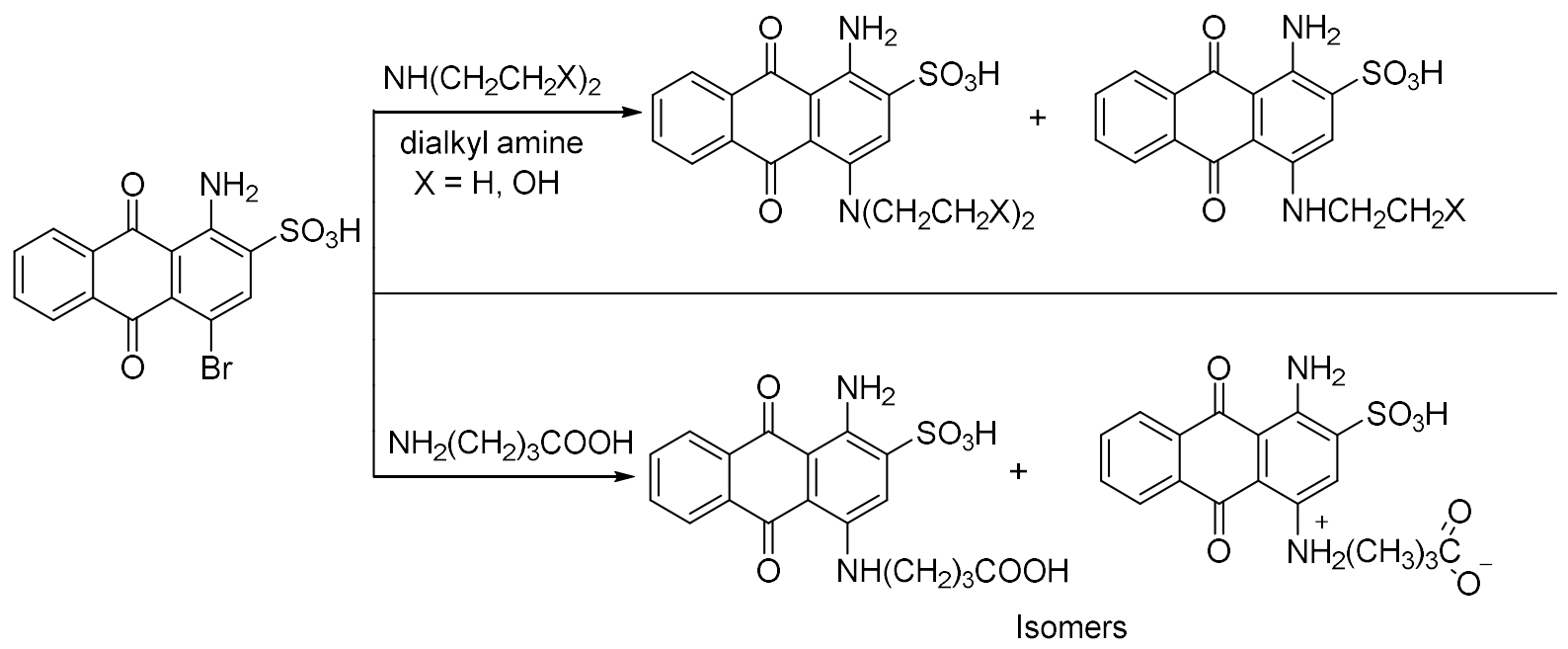

\section{Introduction}

The classical and most widely used strategy for the synthesis of 4-substituted anthraquinone derivatives include the Ullmann coupling reaction [1-4], which involves the treatment of bromaminic acid $\mathbf{1}$, with an amine in the presence of a copper catalyst (Scheme 1) [5-
8]. The reaction typically requires harsh conditions, e.g., high temperatures and long reaction times [9-12]; it suffers from mostly poor yields product 2 [13-15], and the formation of side-products, such as 3 and 4 [16-17]. 


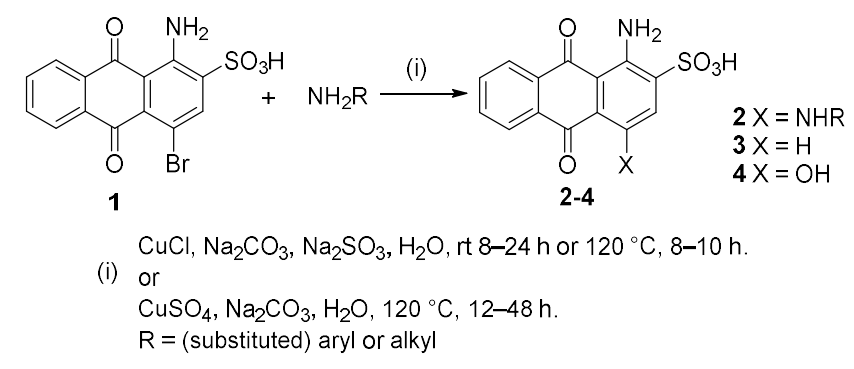

Scheme 1. Classical synthesis of 4-substituted anthraquinone derivatives

Glanzel M. described [18] the anisotropy effect of the aromatic anthraquinone ring $\mathrm{C}, 3-\mathrm{H}-$ proton is protonation by carbon atoms $\mathrm{C}-9$ or $\mathrm{C}$ 10. He also described the effect of the methyl groups rotation of the substituted ring-D, and the fragmentation of the $3-\mathrm{H}$ proton from the structure of the molecule, as shown in Figure 1.
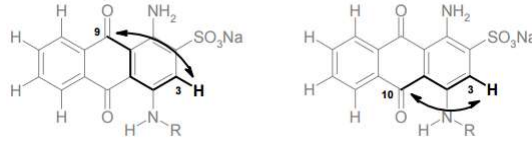

Glanzel M. [18]

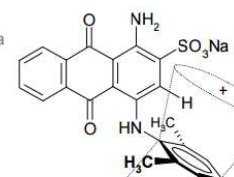

$\mathrm{H}_{3} \mathrm{C}=$
Figure 1. Structures of some 4-substituted 9,10anthraquinone derivatives

Levsen K. [19], described the dissociation of $[\mathrm{M}+\mathrm{H}]^{+}$ions of hydroxylethylamino-anthraquinone 5 (Figure 2), to the $\mathrm{C}-\mathrm{N}$ bond as a result in the elimination of the hydroxyethyl radical $\left({ }^{*} \mathrm{CH}_{2} \mathrm{CH}_{2} \mathrm{OH}\right)$.

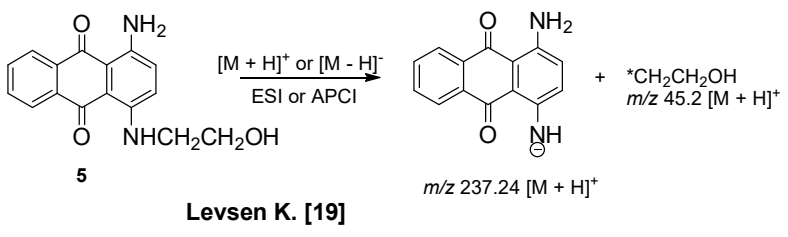

Figure 2. Dissociation 4-substituted 9,10-anthraquinone derivative 5 .
Mass spectrometry is a well recognized highly sensitive characterization method for 4substituted anthraquinone derivatives [20], which we used for our study.

\section{Experimental part}

\section{Material and methods}

All materials purchased from commercial sources and used without purification. Melting points measured in open capillary tubes and uncorrected. The elemental analyses $(\mathrm{C}, \mathrm{H}, \mathrm{N})$ performed using the Perkin-Elmer $2400 \mathrm{CHN}$ analyzer and were within $0.4 \%$ of the theoretical values.

${ }^{1} \mathrm{H},{ }^{13} \mathrm{C}$ NMR spectra were recorded at Varian 400 spectrometer operating at $400 \mathrm{MHz}$ frequency for ${ }^{1} \mathrm{H}$ and $100 \mathrm{MHz}$ for ${ }^{13} \mathrm{C}$ experiments. The peaks were internally referenced to TMS $(0.00 \mathrm{ppm})$ or to the residual undeuterated solvent signal. Peak multiplicities are reported as follows: $\mathrm{s}=$ singlet, br $\mathrm{s}=$ broad singlet, $\mathrm{d}=$ doublet, $\mathrm{t}=$ triplet, $\mathrm{m}=$ multiplet.

The chromatomass spectra were recorded on an Agilent 1100 Series high performance liquid chromatography equipped with a diode matrix with an Agilent LC/MS mass selective detector allowing a fast switching the positive/negative ionization modes. The reaction progress was monitored by the TLC method on Silica gel plates (DC-Fertigfolien ALUGRAM Xtra SILG/UV254, Germany).

\section{Synthesis}


General procedure for preparation of 4substituted-9,10-anthraquinone 14-15, 17-20.

Bromaminic acid $(0.01 \mathrm{~mol})$, was dissolved in $40 \mathrm{ml}$ hot water $\left(70-80{ }^{\circ} \mathrm{C}\right)$, amino derivatives (0.01 mol), acid binding agent sodium bicarbonate $(0.02 \mathrm{~mol})$, copper sulfate $(0.05 \mathrm{~g})$ and ferrous sulfate $(0.05 \mathrm{~g})$ catalysts were then added to it. The reaction mixture stirred and heated to $90{ }^{\circ} \mathrm{C}$. Maintained temperature $90{ }^{\circ} \mathrm{C}$ for $4 \mathrm{~h}$ under stirring. The product salted out by adding sodium chloride, cooled to room temperature, filtered and washed with $10 \% \mathrm{w} / \mathrm{v}$ brine solution.

1-Amino-4-[bis(2-hydroxyethyl)amino]9,10-dioxo-9,10-dihydroanthracene-2-sulfonic acid 14. Blue solid; yield: 46\%, m.p. 295-297 ${ }^{\circ} \mathrm{C}$. LC/MS spectrum, m/z: 405,9 $\quad[\mathrm{M}+\mathrm{H}]^{+}$; $\mathrm{C}_{18} \mathrm{H}_{18} \mathrm{~N}_{2} \mathrm{O}_{7} \mathrm{~S}$; Calculated m/z: 407. ${ }^{1} \mathrm{H}$ NMR (400 MHz, DMSO-d6): $\delta 1.67$ (d, 4H, $\mathrm{NCH}_{2}$ ), 3.67 (d, J = 4.8 Hz, 4H, $\mathrm{OCH}_{2}$ ), 7.49 (br s, 1H, H-3), 7.78 (m, 4H, ArH), 10.14 (s, 1H, OH), 10.79 (br s, 1H, OH); ${ }^{1} \mathrm{H}$ NMR (400 MHz, DMSO-d6 $\left.+\mathrm{CCl}_{4}\right): \delta 1.68\left(\mathrm{~d}, 4 \mathrm{H}, \mathrm{NCH}_{2}\right), 3.48$ (d, 2H, $\mathrm{OCH}_{2}$ ), 3.69 (d, 2H, $\mathrm{OCH}_{2}$ ), 7.38 (br s, 1H, H-3), 7.74 (d, J = 7.6 Hz, 2H, ArH), 7.85 (m, 2H, ArH), 9.35 (s, 1H, OH), 10.14 (br s, 1H, OH). ${ }^{13} \mathrm{C}$ NMR (100 MHz, DMSO-d6): 39.31, 39.5, $39.7\left(\mathrm{CH}_{2}\right) ; 112.8,119.51,120.49,123.55$, 126.22, 129.37, 132.31, 133.19, 133.81, 134.68, 136.37, 137.35 (arom.); 182.00, $184.06(\mathrm{C}=\mathrm{O})$. Anal. Calcd for $\mathrm{C}_{18} \mathrm{H}_{18} \mathrm{~N}_{2} \mathrm{O}_{7} \mathrm{~S}: \mathrm{C}, 53.07$; $\mathrm{H}$ 4.42; N 6.87; S 7.86. Found: C, 53.10; H 4.4; N 6.9; S 7.8 .
1-Amino-4-[(2-hydroxyethyl)amino]-

9,10-dioxo-9,10-dihydroanthracene-2-sulfonic acid 15. ${ }^{1} \mathrm{H}$ NMR (400 MHz, DMSO-d6): $\delta 3.49$ (d, 2H, $\left.\mathrm{CH}_{2}\right), 3.69$ (d, 2H, $\left.\mathrm{CH}_{2}\right), 7.73$ (s, 1H, H3), 7.85 (t, J = 7.7 Hz, 2H, H-6, H-7), 8.25 (d, J = $8 \mathrm{~Hz}, 2 \mathrm{H}, \mathrm{H}-5, \mathrm{H}-8) .{ }^{13} \mathrm{C}$ NMR $(100 \mathrm{MHz}$, DMSO-d6): $45.29 \quad\left(\mathrm{CH}_{2}\right) ; 60.24 \quad\left(\mathrm{CH}_{2}-\mathrm{OH}\right)$; 109.38, 109.67, 121.16, 126.19, 126.36, 132.88, $133.03,134.43,134.47,143.5,143.79,145.84$ (arom.); 181.17, $182.12(\mathrm{C}=\mathrm{O})$. Anal. Calcd for $\mathrm{C}_{18} \mathrm{H}_{18} \mathrm{~N}_{2} \mathrm{O}_{7} \mathrm{~S}:$ C, 50.78; H 4.0; N 7.29; $\mathrm{S}$ 8.04. Found: C, 50.40; H 3.87; N 7.4; S 8.24.

$$
\text { 4-[(4-Amino-9, 10-dioxo-3-sulfo-9, 10- }
$$

dihydroanthracen-1-yl)-amino] butanoic acid 17. Blue solid; yield 56\%, m.p. 301-303 ${ }^{\circ} \mathrm{C}$. LC/MS spectrum: Found, m/z: 405,0 $[\mathrm{M}+\mathrm{H}]^{+}$; $\mathrm{C}_{18} \mathrm{H}_{16} \mathrm{~N}_{2} \mathrm{O}_{7} \mathrm{~S}$; Calculated m/z: 405. ${ }^{1} \mathrm{H}$ NMR (400 MHz, DMSO-d6): $\delta 1.76$ (d, 2H, $\mathrm{NCH}_{2}$ ), 1.91 (d, 2H, $\left.\mathrm{CH}_{2}\right), 2.32\left(\mathrm{~m}, 2 \mathrm{H}, \mathrm{CH}_{2}\right), 2.79$ (s, $\left.2 \mathrm{H}, \mathrm{OCH}_{2}\right), 7.04(\mathrm{~s}, 1 \mathrm{H}, \mathrm{H}-3), 7.78(\mathrm{~s}, 1 \mathrm{H}, \mathrm{ArH})$, 8.04 (s, 1H, ArH), 8.13 (s, 1H, ArH), 8.22 (s, 1H, $\mathrm{ArH}) ;{ }^{1} \mathrm{H}$ NMR (400 MHz, DMSO-d6 $\left.+\mathrm{CCl}_{4}\right): \delta$ 1.82 (d, 2H, $\left.\mathrm{NCH}_{2}\right), 1.98$ (d, 2H, $\left.\mathrm{CH}_{2}\right), 2.32$ (d, $\left.2 \mathrm{H}, \mathrm{CH}_{2}\right), 2.83\left(\mathrm{~d}, 2 \mathrm{H}, \mathrm{CH}_{2}\right), 7.2$ (br s, 1H, H-3), 7.73 (d, 2H, ArH), 8.05 (d, J = 8 Hz, 2H, ArH). ${ }^{13} \mathrm{C}$ NMR (100 MHz, DMSO-d6): 40.14, 42.24, 49.47, 53.22 $\left(\mathrm{CH}_{2}\right)$; 108.23, 113.48, 125.18, 125.9, 133.05, 134.73, 140.98, 143.98, 144.34, 145.34, 148.43, 154.95 (arom.); 181.43, 186.69 $(\mathrm{C}=\mathrm{O})$. Anal. Calcd for $\mathrm{C}_{18} \mathrm{H}_{16} \mathrm{~N}_{2} \mathrm{O}_{7} \mathrm{~S}: \mathrm{C}, 53.33$; H 3.95; N 6.91; S 7.9. Found: C, 53.3; H 3.9; N $6.9 ; \mathrm{S} 8.1$. 
1-Amino-4-(1H-imidazol-1-yl)-9,10-

dioxo-9,10-dihydroanthracene-2-sulfonic acid

18. Yellow solid; Recr. from aceton yield $100 \%$, m.p. $255-257^{\circ} \mathrm{C}$. LC/MS spectrum: Found, m/z: $370,2[\mathrm{M}+\mathrm{H}]^{+} ; \mathrm{C}_{17} \mathrm{H}_{11} \mathrm{~N}_{3} \mathrm{O}_{5} \mathrm{~S} ;$ Calculated $\mathrm{m} / \mathrm{z}$ : 370. ${ }^{1} \mathrm{H}$ NMR (400 MHz, DMSO-d6): $\delta 7.8$ (m, $\mathrm{J}=8 \mathrm{~Hz}, 8 \mathrm{H}, \mathrm{ArH}), 9.53\left(\mathrm{~s}, 1 \mathrm{H}, \mathrm{SO}_{3} \mathrm{H}\right)$. Anal. Calcd for $\mathrm{C}_{17} \mathrm{H}_{11} \mathrm{~N}_{3} \mathrm{O}_{5} \mathrm{~S}: \mathrm{C}, 55.13 ; \mathrm{H} 2.97 ; \mathrm{N}$ 11.35; S 8.65. Found: C, 55.10; H 2.9; N 11.37; S 8.63 .

1-Amino-9,10-dioxo-4-(2-oxo-4-sulfanylidene-1,3-thiazolidin-3-yl)-9,10-dihydroanthracene-2-sulfonic acid 19. Blue solid; Yield 14.3\%, m.p. $285-288^{\circ} \mathrm{C}$. LC/MS spectrum: Found, m/z: 435,2 [M+H] $]^{+} ; \mathrm{C}_{17} \mathrm{H}_{11} \mathrm{~N}_{3} \mathrm{O}_{5} \mathrm{~S}$; Calculated m/z: 434. ${ }^{1} \mathrm{H}$ NMR (400 MHz, DMSO-d6): $\delta 3.32$ (d, $\left.2 \mathrm{H}, \mathrm{CH}_{2}\right), 7.26(\mathrm{~s}, 1 \mathrm{H}, \mathrm{H}-3), 7.82(\mathrm{~d}, \mathrm{~J}=7.2 \mathrm{~Hz}$, 2H, H-6, H-7), 8.33 (d, J = 8 Hz, 2H, H-5, H-8). Anal. Calcd for $\mathrm{C}_{17} \mathrm{H}_{10} \mathrm{~N}_{2} \mathrm{O}_{6} \mathrm{~S}_{3}$ : C, 46.89; S 22.06. Found: C, 46.85; S 22.00.

\section{1-Amino-4-[(pyridin-3-yl)amino]-9,10-} dioxo-9,10-dihydroanthracene-2-sulfonic acid 20. Blue solid; Yield $15 \%$, m.p. $250-251{ }^{\circ} \mathrm{C}$. LC/MS spectrum: Found, m/z: 394,2 [M+H] $]^{+}$; $\mathrm{C}_{19} \mathrm{H}_{13} \mathrm{~N}_{3} \mathrm{O}_{5} \mathrm{~S}$; Calculated m/z: 395. ${ }^{1} \mathrm{H}$ NMR (400 MHz, DMSO-d6): $\delta 7.62$ (s, 1H, H-3), 7.7 (d, 2H, H-5, H-7), 7.85 (t, J = 6.4 Hz, 3H, ArH), $8.08(\mathrm{~d}, \mathrm{~J}=6.4 \mathrm{~Hz}, 2 \mathrm{H}, \mathrm{H}-5, \mathrm{H}-8) ;{ }^{1} \mathrm{H}$ NMR (400 MHz, DMSO-d6 $+\mathrm{CCl}_{4}$ ): $\delta 7.63$ (br s, $1 \mathrm{H}$, H-3), 7.84 (t, J = 6.8 Hz, 2H, H-6, H-7), 7.96 (s, $1 \mathrm{H}, \mathrm{ArH}), 8.00$ (s, 1H, ArH), 8.10 (s, 1H, ArH), 8.17 (d, J = 6.8 Hz, 2H, H-5, H-8). ${ }^{13} \mathrm{C}$ NMR (100 MHz, DMSO-d6): 122.58, 124.42, 127.11,
$127.40,131.71,133.22,134.83,135.58,136.29$, 140.89, 152.98, 173.58 (arom.); 180.82, 181.09 $(\mathrm{C}=\mathrm{O})$. Anal. Calcd for $\mathrm{C}_{19} \mathrm{H}_{13} \mathrm{~N}_{3} \mathrm{O}_{5} \mathrm{~S}: \mathrm{C}, 57.73$; H 3.29; N 10.63; S 8.1. Found: C, 57.7; H 3.3; N $10.7 ; \mathrm{S} 8.17$.

\section{Results and discussion}

Substitution with dialkyl amines 6-7 was starting according to the procedure described previously [21]. However, the desirable products were not obtained. In the case of nucleophilic substitution on diethanolamine (7) formed product $15-37 \%$ on the LC-MS $(\mathrm{m} / z 363.2$ $\left.[\mathrm{M}+\mathrm{H}]^{+}\right)$. In the case of substitution brom on diethylamine (6), formations of side-product (4) was $28 \%\left(\mathrm{~m} / z 302.0[\mathrm{M}+\mathrm{H}]^{-}\right)$. Then we used the general procedure (experimental data) for nucleophilic substitution in bromaminic acid (Scheme 3). In the case of substitution brom of diethanolamine (7), the product 15 was still present in LC-MS $\left(m / z 364.0[\mathrm{M}+\mathrm{H}]^{+}\right)$. The ${ }^{13} \mathrm{C}$ NMR spectra characteristic signals carbon $\left(\mathrm{CH}_{2}-\mathrm{CH}_{2}-\mathrm{OH}\right)$ was 45.29 and $60.24 \mathrm{ppm}$; the resonance signals for two carbonyl carbons 1amino-4-[(2-hydroxyethyl)amino]-9,10-dioxo9,10-dihydro-anthracene-2-sulfonic acid (15) at $\delta 181.17$ and $182.12 \mathrm{ppm}$. Ullmann coupling reaction of bromaminic acid with diethylamine (6), leads to the formation product $13(\mathrm{~m} / \mathrm{z} 347.0$ $\left.[\mathrm{M}+\mathrm{H}]^{+}\right)$, it was fixed using diode array detection (DAD).

The desired 4-diethanol-substituted product 14, separated by HPLC. ${ }^{1} \mathrm{H}$ NMR is 
present in 8 aliphatic protons of methylene groups in solvents (DMSO-d6) and (DMSO$\mathrm{d} 6+\mathrm{CCl}_{4}$ ), characteristic shifts of 5 aromatic protons at 7.38-8.24 ppm, respectively.

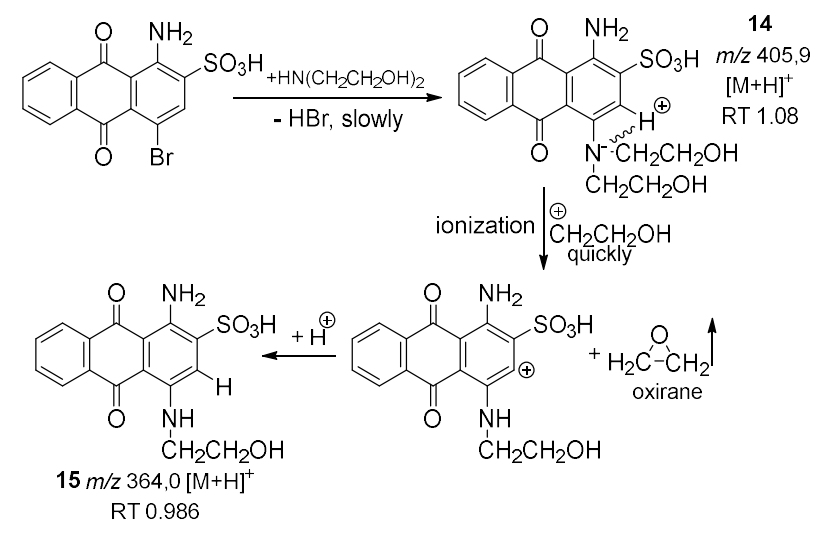

Figure 3. Proposed mechanism

The proposed mechanism for the formation of compound $15\left(\mathrm{~m} / \mathrm{z} 364.0[\mathrm{M}+\mathrm{H}]^{+}\right)$ (Figure 2), depicted in the LC-MS spectrum (Scheme 2). And explains fragmentation 3-Hproton of the ethylhydroxyl radical which transformed into oxyran.
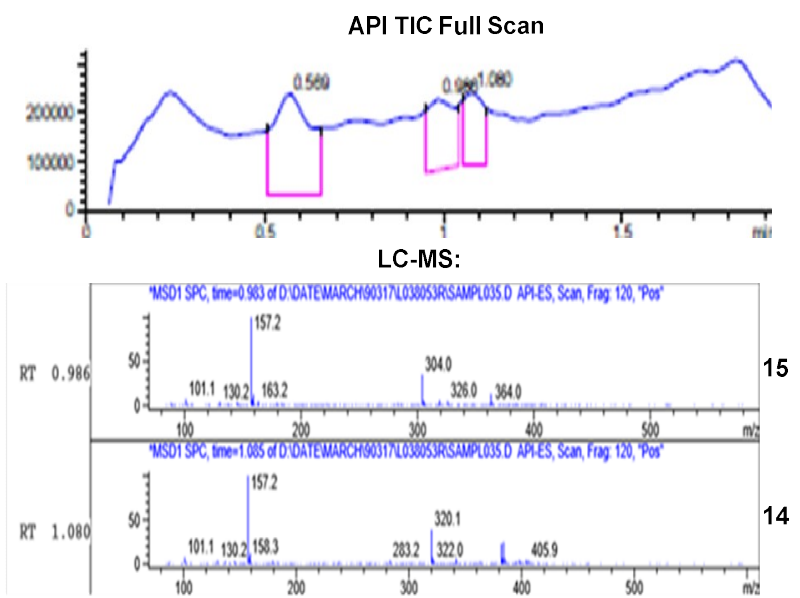

Scheme 2. Chromatomass-spectra substitution by amino acids

In case substitution brom of glycine $\mathbf{8}$, is formation of side-products 4 - 38.9\% ( $/ \mathrm{m} / \mathrm{z} 318.2$ $\left.[\mathrm{M}+\mathrm{H}]^{-}\right)$and 3 was $24.5 \%\left(\mathrm{~m} / \mathrm{z} 302.0[\mathrm{M}+\mathrm{H}]^{-}\right)$. The poor yields of product $\mathbf{1 6}$ explained by the weak base of aminoacetic acid (8). Reaction of bromaminic acid with amino acid 9, the formation of isomers $\mathbf{1 7}\left(\mathrm{m} / \mathrm{z} 405.0[\mathrm{M}+\mathrm{H}]^{+}\right)$.

After purification by silica gel flash column chromatography, eluted with ethyl acetate:aceton 1:3 v/v, compound $\mathbf{1 7}$ was obtained. The ${ }^{1} \mathrm{H}$ NMR spectrum in (DMSO-d6) there are 6 aliphatic $\mathrm{CH}_{2}$ methylene protons in the region at $1.76-2.79 \mathrm{ppm}$. The ${ }^{13} \mathrm{C} \mathrm{NMR}$ spectra was characteristic signals carbon methylene groups in the region at 40.14$53.22 \mathrm{ppm}$.

substitution by heterocyclic amines

The yield of product $\mathbf{1 8}$ after recrystallization with acetone was $100 \%(\mathrm{~m} / \mathrm{z}$ $\left.370.2[\mathrm{M}+\mathrm{H}]^{+}\right)$. The aromatic protons were visible as a multiplet of $\delta$ 7.7-7.8 $\mathrm{ppm}$. In the case of substitution bromaminic acid with 3aminopyridine (12), the yield of the main product 20 was $14.9 \%\left(m / z 394.0[\mathrm{M}+\mathrm{H}]^{+}\right)$the ${ }^{1} \mathrm{H}$ NMR spectrum nine aromatic protons appeared in the region of 7.62-8.24 ppm (DMSO-d6) and 7.63$8.17\left(\mathrm{DMSO}-d 6+\mathrm{CCl}_{4}\right)$. 
<smiles>Nc1c(S(=O)(=O)O)cc(NCC(=O)O)c2c1C(=O)c1ccccc1C2=O</smiles><smiles>CCNc1cc(S(=O)(=O)[18F])c(N)c2c1C(=O)c1ccccc1C2=O</smiles>
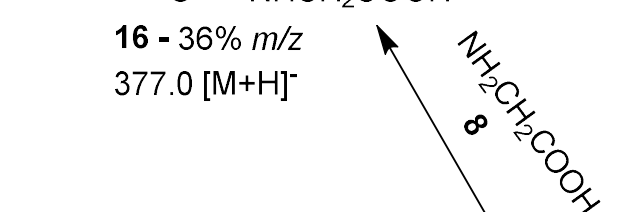

$\mathrm{O} \quad \mathrm{NH}\left(\mathrm{CH}_{2}\right)_{3} \mathrm{COOH}$<smiles>[R]c1cc([Se])c(N)c2c1C(=O)c1ccccc1C2=O</smiles>

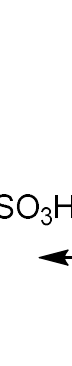<smiles>OCCNCCO</smiles>

$3 \mathrm{R}_{1}=\mathrm{H}-31 \%\left(\mathrm{~m} / \mathrm{z} 302.2[\mathrm{M}+\mathrm{H}]^{+}\right)$

$14 \mathrm{R}_{1}=\mathrm{N}\left(\mathrm{CH}_{2} \mathrm{CH}_{2} \mathrm{OH}\right)_{2}-46 \%(\mathrm{~m} / \mathrm{z}$ $\left.405.9[\mathrm{M}+\mathrm{H}]^{+}\right)$

$15 \mathrm{R}_{1}=\mathrm{NHCH}_{2} \mathrm{CH}_{2} \mathrm{OH}-17 \%(\mathrm{~m} / \mathrm{z}$ $\left.364.0[\mathrm{M}+\mathrm{H}]^{+}\right)$

$$
\begin{aligned}
& 3 \mathrm{R}=\mathrm{H}-47 \%\left(\mathrm{~m} / \mathrm{z} 302.2[\mathrm{M}+\mathrm{H}]^{-}\right) \\
& \begin{array}{l}
\mathrm{R}=\mathrm{OH}-23 \%\left(\mathrm{~m} / \mathrm{z} 318.0[\mathrm{M}+\mathrm{H}]^{+}\right) \\
13 \mathrm{R}=\mathrm{NHCH} \mathrm{CH}_{3}-29 \%\left(\mathrm{~m} / \mathrm{z} 347.0[\mathrm{M}+\mathrm{H}]^{+}\right)
\end{array}
\end{aligned}
$$

$18-100 \% \mathrm{~m} / \mathrm{z} 370.2[\mathrm{M}+\mathrm{H}]^{+}$<smiles>Nc1c(S(=O)(=O)O)cc(-n2ccnc2)c2c1C(=O)c1ccccc1C2=O</smiles>

10

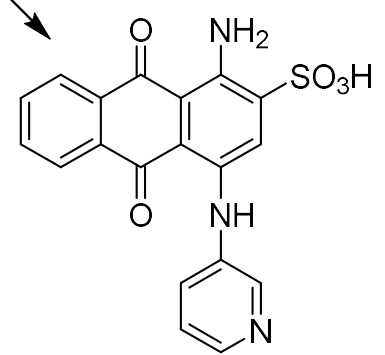

$20-15 \% \mathrm{~m} / \mathrm{z} 394.2[\mathrm{M}+\mathrm{H}]^{+}$

Scheme 3. Synthesis of 4-substituted 9,10-anthraquinonsulfonic acids

hydroxyethyl group $\left({ }^{*} \mathrm{CH}_{2} \mathrm{CH}_{2} \mathrm{OH}\right)$ or ethyl

The main undesirable product identified in all reactions was 1-amino-4-hydroxy-9,10dioxo-9,10-dihydroanthracene 2-sulfonate (4 Scheme 3) formed by attack of the competing nucleophile hydroxide.

\section{Conclusions}

Fragmentation mechanisms of protonated 4-substituted-9,10-anthraquinones and its derivatives investigated by atmospheric pressure ionization with mass spectrometry (MS). The major fragmentation pathways were loss of the group $\left({ }^{*} \mathrm{CH}_{2} \mathrm{CH}_{3}\right)$ from fourth position of the $\mathbf{C}$ ring. Determined LC/MS that as a result of nucleophilic substitution of brom on $\gamma$ aminobutyric acid, the final products are the isomers of compound 17.

\section{Acknowledgements}

The work was carried out with the financial support of the Ministry of Education and Science of Ukraine (Project No. 0119U103131). 


\section{References}

[1] Ullmann F.; Bielecki J. Ueber Synthesen in der Biphenylreihe". Chemische Berichte., 1901, 34(2): 21742185. doi:10.1002/cber.190103402141

[2] Jiao, J.; Zhang, X.-R.; Chang, N.-H.; et.al. A facile and practical copper powder-catalyzed, organic solvent-and ligand-free Ulmann amination of aryl halides. J. Org. Chem. 2011, 76:1180-1183; DOI: $10.1021 /$ jo102169t

[3] Ma, D.W.; Cai, Q.; Copper/amino acid catalyzed cross-couplings of aryl and vinyl halides with nucleophiles Acc. Chem. Res., 2008, 41:1450-1460; DOI: 10.1021/ar8000298

[4] Carril, M.; Martin, R.S.; Dominguez, E.; Palladium and copper-catalysed arylation reactions in the presence of water, with a focus on carbon-heteroatom bond formation. Chem. Soc. Rev., 2008, 37:639 - 647; DOI: 10.1039/B709565C

[5] Patel, N.B.; Patel, A.L.; Characterization, application and microbial study of imidazole base acid antraquinone dyes. Oriental J. of Chem., 2008, 24:551558.

[6] Glanzel, M.; Bultmann, R.; Starke, K.; Frahm, A.W. Constitunal isomer of Reactive Blue 2 - selective P2Y-receptor antagonists ? Eur. J. Med. Chem. 2003, 38:303-312.

[7] Glanzel, M.; Bultmann, R.; Starke, K.; Frahm, A.W. Structure-activity relationships of novel P2-receptor antagonists structurally related to Reactive Blue 2. Eur. J. Med. Chem. 2005, 40:1262-1276.

[8] Baqi Y.; Muller C.E. Synthesis of alkyl- and aryl-amino-substituted anthraquinone derivatives by microwave-assisted copper (0)-catalyzed Ullmann coupling reactions. Nature Prot. 2010, 5:387-390; DOI: 10.1038 /nprot.2010.63

[9] Lee, C.-C.; Chen, C.-L.; Liu, F.-L.; et. al. Development of 1-Amino-4-(phenylamino)anthraquinone-2-sulfonate Sodium Derivatives as a New Class of Inhibitors of RANKL-Induced Osteoclastogenesis. Arch.
Pharm. Chem. Life Sci., 2016, 349:342-355. DOI: 10.1002/ardp.201500475

[10] Baqi Y.; Weyler S.; Iqbal J.; Muller C.E. Strcture-activity relationships of anthraquinone derivatives derived from bromaminic acid as inhibitors of ectonucleoside triphosphate diphosphodydrolases (ENTPDases). Purinergic Signalling. 2009, 5:91-106; DOI: 10.1007/s1 1302-008-9103-5

[11] Baqi Y.; Malik E.; Rashed M.; Wingen L.; Muller C.E. Ullmann reactions of 1-amino-4bromoantraquinone bearing various 2-substituents furnishing novel dyes. Dyes and Pigments. 2016, 131:3340; DOI: 10.1016/j.dyepig.2016.03.023

[12] Baqi Y.; Malik E.; Muller C.E. Syntheses of 2-substituted 1-amino-4-bromoanthraquinones (bromaminic acid analogues) - precursors for dyes and drugs. Beilstein J. Org. Chem., 2015, 11:2326-2333; DOI: 10.3762/bjoc. 11.253

[13] Roy S.; Large R.J.; Akande A.M.; et.al. Development of GoSlo-SR-5-69, a potent activator of large conductance $\mathrm{Ca}^{2+}$-activated $\mathrm{K}^{+}(\mathrm{BK})$ channels. Eur. J. Med. Chem., 2014, 75:426-437; DOI: org/10.1016/j.ejmech.2014.01.035

[14] Olubunmi, A.; Olatunji A. Gabriel, Condensation and Cellulosic Derivatization of Bromamine Acid. The Pacific Journal of Science and Technology. 2009, 10:788-794.

[15] Yildiz, E.; Keles, M.; Kaya, A.; Dincer, S. Mononuclear Fe (III), Cr (III), Co (II) Metal Complexes Based on Azo-Anthraquinone Moieties: Synthesis, Characterizations and Antibacterial Activities. Chem. Sci. Trans., 2013, 2(2):547-555.

[16] Baqi Y.; Muller C.E. Rapid and efficient microwave-assisted copper (0)-catalyzed Ullmann coupling reaction. Org. Lett. 2007, 9:1271-1274; DOI: $10.1021 / \mathrm{ol} 070102 \mathrm{v}$

[17] Vrba, Z.; Mechanism of the Ullmann condensation. Chem. Commun, 1981, 46:92 - 100.

[18] Glanzel M. Synthesis P2-PezeptorAntagonistenin der Reiheder Reaktivblau 2 Farbstobbe 
und deren Struktur-Wirkung Beziehungen: dissertation zur Erlangung der Doktorwürde. 2003.

[19] Levsen K.; Schiebel H.; Terlouw J. Evenelectron ions: a systematic study of the neutral species lost in the dissociation of quasi-molecular ions. J. Mass Spektrom. 2007, 42:1028 - 1033. DOI: 10.1002/jms. 1234

[20] Enaud, E.; Trovaslet, M.; Bruvneel, F.; et. al., A novel azoanthraquinone dye made through innovative enzymatic process. Dyes and Pigments., 2010, 85(3):102. DOI 10.1016/j.dyepig.2009.10.010

[21] Inoue, H.; Nakamura, K.; Kato, S.; Hida, M. The Nucleophilic Photo-substitution reaction of anthraquinone derivatives. II. The Mechanism of the Photoamination of sodium 1-amino-4-bromanthraquinone2-sulfate. J. Chem. Soc., of Japan., 1975, 48(10):2872 2876. 